\title{
La velocidad de aumento del PSA permite identificar pacientes con riesgo de muerte post prostatectomia radical
}

\section{Objetivo}

Valorar la utilidad de la velocidad de aumento del PSA durante el año previo a la cirugía como factor pronostico de mortalidad.

Diseño

Estudio retrospectivo de cohortes.

\section{Lugar}

Base de datos de pacientes prostatectomizados del Barnes-Jewish Hospital in St Luis. Estados Unidos.

\section{Pacientes}

Se analizaron 1095 pacientes prostatectomizados entre 1989 y 2002. Se excluyeron 20 pacientes con tratamientos adyuvantes y 689 pacientes con una sola determinación del PSA previa a la cirugía. La mediana de seguimiento fue de 5.1 años con rango entre 0.5 y 13.1 años.

\section{Evaluación de los factores pronósticos}

Se evaluaron múltiples factores pronósticos como el score de Gleason, el estadio tumoral T, PSA. y la velocidad de aumento del PSA, estimando la recurrencia de enfermedad, muerte por cualquier causa y muerte por cáncer de próstata.

\section{Medición de resultado principal}

El análisis de los datos de los 1095 pacientes estudiados permitió estratificarlos en cuatro categorías de acuerdo a la velocidad de aumento del PSA dentro del año previo a la cirugía.

\section{Resultados principales}

Una velocidad de aumento anual del PSA mayor a $2 \mathrm{ng} / \mathrm{ml}$ comparada con una velocidad menor a $2 \mathrm{ng} / \mathrm{ml}$ se asoció significativamente a una sobrevida mas corta, muerte por cáncer de próstata $(P<0,001)$ y muerte por otra causa $(P=0,01)$. Un $P S A$ elevado en el momento del diagnóstico $(\mathrm{P}=0,01)$, score de Gleason 8, 9 o 10 $(P=0,02)$, y un estadio clínico T2 $(P<0,001)$ también predicen el tiempo de muerte por cáncer de próstata.

\section{Conclusiones}

Aquellos pacientes cuyos niveles de PSA aumentan mas de 2 $\mathrm{ng} / \mathrm{ml}$ durante el año previo al diagnostico de cáncer de próstata tienen mayor riesgo de morir por la enfermedad a pesar de realizarse una prostatectomia radical, que en aquellos en que el nivel de PSA es menor.

\section{Comentario}

A pesar de una correcta estratificación, entre el 15 y $35 \%$ de los pacientes $(50.000$ pac.) prostatectomizados recaerán anualmente en los EEUU ${ }^{1}$. Sabemos que muchos de ellos tendrán una evolución lenta y solo algunos morirán a causa de la progresión de la enfermedad. Sería de gran utilidad poder inferir quienes tienen más riesgo de morir por cáncer de próstata a pesar de una correcta estatificación y tratamiento.

Datos como el PSA, Gleason y la estratificación han demostrado ser útiles en la identificación de pacientes con mayor riesgo de progresión. Las modificaciones de los valores de PSA en el tiempo, como es la velocidad de aumento, también sirven a este efecto, inclusive son de utilidad para inferir el sitio de recaída post tratamiento ${ }^{2}$. En este caso para determinar si el valor de la velocidad de aumento del PSA es útil para detectar pacientes con riesgo de muerte, los autores estratifican los datos de velocidad de aumento del PSA obtenidos durante el año previo a la cirugía y lo relacionan con los porcentajes de recurrencia y muerte por cáncer de próstata. Observan que los pacientes que se encuentran en el cuartilo $>2 \mathrm{ng} / \mathrm{ml} /$ año tienen un aumento significativo del riesgo de morir por cáncer de próstata comparado con los pacientes que presentaron una velocidad menor de aumento del PSA. No es una observación menor teniendo en cuenta que el 24\% de los 1095 pacientes evaluados se encontraron dentro del grupo de riesgo.
En el presente trabajo, el $43 \%$ de los pacientes tenia PSA igual o menor a $4 \mathrm{ng} / \mathrm{ml}$. En nuestra práctica diaria es raro que a un paciente con una primera determinación de PSA normal se le repita otra antes del año, salvo valores en el límite de lo normal o antecedentes familiares de muerte por cáncer de próstata. Además muchos de nuestros pacientes son referidos y no contamos con determinaciones anteriores de PSA. Por lo tanto es raro que en nuestra población de pacientes con cáncer de próstata localizado podamos hacer estas observaciones. Si con el tiempo estos datos son confirmados, los urólogos podrían adecuar tratamientos agresivos a pacientes con tumores con mayor riesgo de muerte. Además en estos casos se podrían ensayar protocolos de adyuvancia. Para los médicos clínicos, el trabajo refuerza la idea de prestar atención a aumentos del PSA en los pacientes que se encuentran bajo su control.

\section{Conclusión del comentador}

Como conclusión el trabajo logra el objetivo planteado analizando la velocidad de aumento del PSA como factor de mal pronóstico y mayor riesgo de muerte por cáncer de próstata.

\section{Dr. Carlos Giudice (h) [ Servicio de Urología, Hospital Italiano Buenos Aires.]}

Giudice, C. La velocidad de aumento del PSA permite identificar pacientes con riesgo de muerte post prostatectomia radical. Evid. actual. práct. ambul. 2004;7:138. Comentado de: Preoperative PSA Velocity and the Risk of Death from Prostate Cancer after Radical Prostatectomy. D'Amico A.; Ming-Hui Chen; Roehl K.A. and Catalona W.J. NEW Engl J Med. 2004; 351: 125-5.

\section{Referencias}

1.Pound CR, Partin AW, Eisenberger MA, et al. Natural history of progression after PSA elevation following radical prostatectomy. JAMA 1999 May 5; $281(17)$ : $1591-7$.

2.Roberts SG, Blute ML, Bergstralh EJ, et al. PSA doubling time as a predictor of clinical progression after biochemical failure following radical prostatectomy for prostate cancer. Mayo Clin Proc 2001 Jun; 76(6): 576-81. 